\title{
Temperature dependence of spectral functions for the one-dimensional Hubbard model: comparison with experiments
}

\author{
A. Abendschein ${ }^{(1),(2)}$ and F. F. Assaad ${ }^{(1)}$ \\ (1) Institut für theoretische Physik und Astrophysik, \\ Universität Würzburg, Am Hubland D-97074 Würzburg, Germany \\ (2) Laboratoire de Physique Théorique, IRSAMC, \\ Université Paul Sabatier, 118, route de Narbonne,31062 Toulouse, France
}

\begin{abstract}
We study the temperature dependence of the single particle spectral function as well as of the dynamical spin and charge structure factors for the one-dimensional Hubbard model using the finite temperature auxiliary field quantum Monte Carlo algorithm. The parameters of our simulations are chosen so to at best describe the low temperature photoemission spectra of the organic conductor TTF-TCNQ. Defining a magnetic energy scale, $T_{J}$, which marks the onset of short ranged $2 k_{f}$ magnetic fluctuations, we conclude that for temperatures $T<T_{J}$ the ground state features of the single particle spectral function are apparent in the finite temperature data. Above $T_{J}$ spectral weight transfer over a scale set by the hopping $t$ is observed. In contrast, photoemission data points to a lower energy scale below which spectral weight transfer occurs. Discrepancies between Hubbard model calculations and experiments are discussed.
\end{abstract}

PACS numbers: 71.27.+a, 71.10.-w, 71.10.Fd

\section{INTRODUCTION}

The hallmark of Luttinger liquids lies in spin-charge separation; an electron fractionalizes into a spinon carrying the spin degrees of freedom and a holon carrying the charge. Detecting spin-charge separation relies on the study of the single particle spectral function. From the theoretical point of view, the ground state low energy properties of the single particle spectral function can be obtained from bosonization [1]. This approach yields two branch cuts, corresponding to the spinon and the holon, dispersing linearly from the Fermi wave vector with spin and charge velocities. Beyond this low energy limit, exact calculations of the spectral function have been carried out for the infinite $U$ Hubbard model [2]. Taking into account that in this limit the spin velocity vanishes, the results stand in agreement with the low energy bosonization picture. Furthermore the calculations reveal higher energy features such as a holon shadow band. Hence, distinct signatures of Luttinger liquids may be found in a wide energy range thus facilitating detection in photoemission experiments. Beyond the infinite $U$ limit, numerical simulations such as dynamical DMRG (DDRMG) 3 or Quantum Monte Carlo [4] can be used to investigate the zero temperature properties of the spectral function. In particular, $T=0$ DDMRG results for the Hubbard model have been compared successfully with low temperature, $T=60 \mathrm{~K}$, photoemission experiments on the organic one-dimensional conductor TTF-TCNQ 5]. In the temperature range $60 K<T<260 K$ experiments point towards substantial spectral weight transfer. Keeping the model parameters which reproduce the low temperature data, our aim is to understand if the experimentally observed temperature behavior of the spectral function can be reproduced by finite temperature model calculations.
The Hubbard model we consider reads:

$$
\begin{aligned}
H=- & t \sum_{\langle i, j\rangle, \sigma}\left(c_{i \sigma}^{\dagger} c_{j \sigma}+\text { h.c. }\right)+ \\
& +U \sum_{i} n_{i \uparrow} n_{i \downarrow}-\mu \sum_{i}\left(n_{i \uparrow}+n_{i \downarrow}\right)
\end{aligned}
$$

where $t$ it the hopping amplitude, $U$ the Coulomb repulsion, $\mu$ the chemical potential, and the first sum runs over nearest neighbors. $c_{i \sigma}^{\dagger}\left(c_{i \sigma}\right)$ creates (annihilates) an electron in the Wanier state centered around lattice site $i$ and with $z$-component of spin $\sigma=\uparrow, \downarrow$. Comparison between DDMRG results and experiments point to a parameter set $U / t=4.9, t=0.4 \mathrm{eV}$ and $n=0.59$ for an adequate description of the TCNQ chain. Throughout this article we will keep those parameters fixed and vary the temperature. The organization and main results of the paper are as follows. In section 【 we briefly present the finite temperature auxiliary field quantum Monte Carlo (QMC) method and the maximum entropy method we have used to analytically continue the imaginary time QMC data. Section III is dedicated to the results. To map out the scales involved in the problem, we first consider the temperature dependence of the spin and charge susceptibilities as well as of the spin and charge dynamical structure factors. This allows us to define a magnetic crossover energy scale, $T_{J}$, below which the magnetic $2 k_{f}$ correlation length increases substantially as a function of decreasing temperature. In section IIIB we analyze the temperature dependence of the single particle spectral function and arrive to the conclusion that $T_{J}$ is the only low energy scale at hand in the problem. That is, for temperatures below $T_{J}$ the overall features of the zero temperature spectral function are well reproduced. Above $T_{J}$ we observe spectral weight transfer over energy scale set by $t$. With $t=0.4 \mathrm{eV}$ our estimated value of $T_{J}$ is $T_{J} \simeq 400 \mathrm{~K}$. In the conclusion (Sec. IV), we 


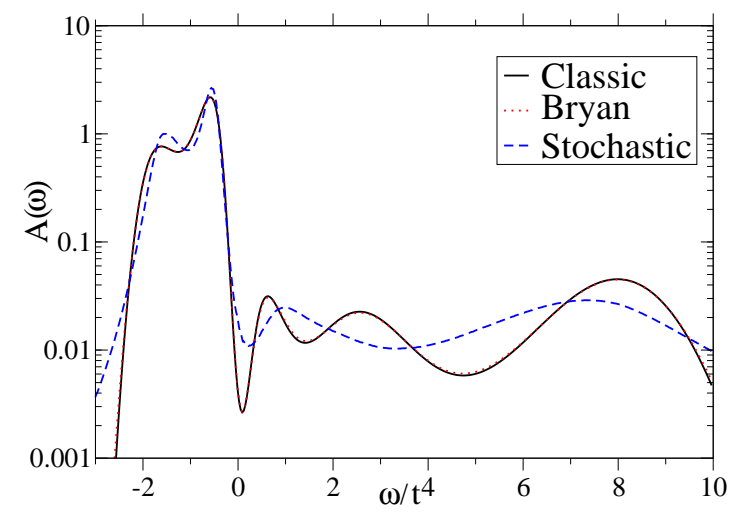

FIG. 1: Comparison of different Maximum Entropy methods for the spectral function at $k=0$, of the one-dimensional Hubbard model at $U / t=4.9, n=0.59$ and $\beta t=7$. Note the semi-log scale.
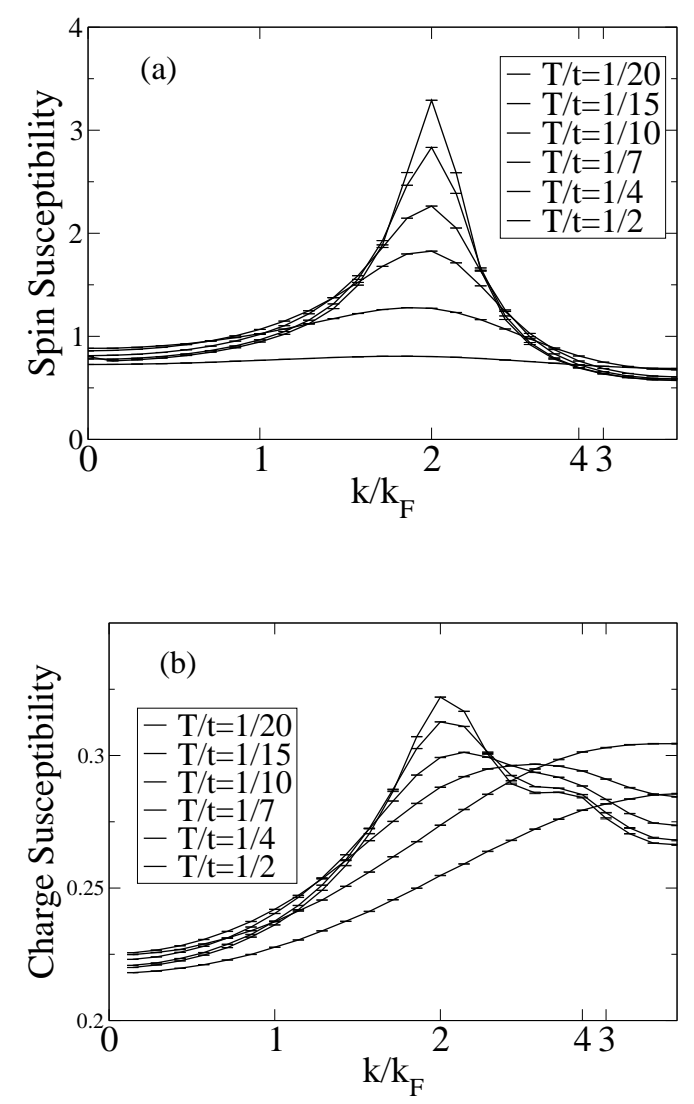

FIG. 2: Spin and charge static susceptibilities, $\chi_{c}(k, \omega=0)$, as a function of wave vector. At $2 k_{f}$ the different lines from bottom to top correspond to the temperatures $\beta t=$ $2,4,7,10,15,20$

discuss possible scenarios to understand the discrepancy between experimental data and model calculations.

\section{QUANTUM MONTE CARLO ALGORITHM}

We have used a generic implementation of the finite temperature grand canonical auxiliary field algorithm [6] to compute imaginary time displaced Green functions, $G(\vec{k}, \tau)=\left\langle c_{\vec{k}}(\tau) c_{\vec{k}}^{\dagger}(0)\right\rangle$, as a function of temperature for the one-dimensional Hubbard model on a 48-site chain with periodic boundary conditions. The spectral function is extracted from the imaginary time data by inverting the equation:

$$
\begin{aligned}
& \left\langle c_{\vec{k}}(\tau) c_{\vec{k}}^{\dagger}(0)\right\rangle=\int \mathrm{d} \omega K(\tau, \omega) A(\vec{k}, \omega) \text { with } \\
& K(\tau, \omega)=\frac{1}{\pi} \frac{e^{-\tau \omega}}{1+e^{-\beta \omega}} .
\end{aligned}
$$

Since this inversion is numerically ill defined we have used the Maximum Entropy method and favored a recently proposed stochastic version [7]. The stochastic formulation has the appealing property that it formally contains the generic Maximum Entropy method (MEM) 8, 2] at the mean field level. In the generic MEM, there is no free parameter. In particular, $\alpha$ which determines how much information is taken from the default model is determined self-consistently. In contrast, in the stochastic approach, there is no sharp way of determining $\alpha$ and we have used the criterion proposed by K. Beach [7]. In Fig. 11 we compare the different Maximum Entropy methods for the one-dimensional Hubbard model at $U / t=4.9$, $n=0.59$ and $\beta t=7$. The Brayn and Classic Maximum entropy formulations [8] yield identical spectral function. The two peak structure at $\omega / t<0$ corresponding to the holon and spinon branches, is sharper in the stochastic approach. At $\omega / t>0$ the stochastic spectrum shows less features than the Classic Maximum Entropy method. It is know that the Classic Maximum Entropy has difficulties in reproducing flat spectra and generates a curve oscillating smoothly around the correct value. This problem seems to be alleviated by the stochastic approach. Hence, our overall opinion is that the stochastic approach does better at reproducing sharp features as well as flat regions in the spectra. Finally we note that we have always taken the covariance matrix into account.

\section{RESULTS}

We compute the single particle spectral function as well as the dynamical spin and charge structure factors as a function of temperature. We choose a parameter set which at best describes the low temperature properties of the photoemission spectra of TTF-TCNQ [5]. That is for the TCNQ band, $U / t=4.9, t=0.4 \mathrm{eV}$ and a filling fraction $n=0.59$. We cover the following range of inverse temperatures $\beta t=2,4,7,10,15$ and $\beta t=20$. Below, we will first discuss two particle properties so as to pin down scales and then consider the temperature behavior of the single particle spectral function. 

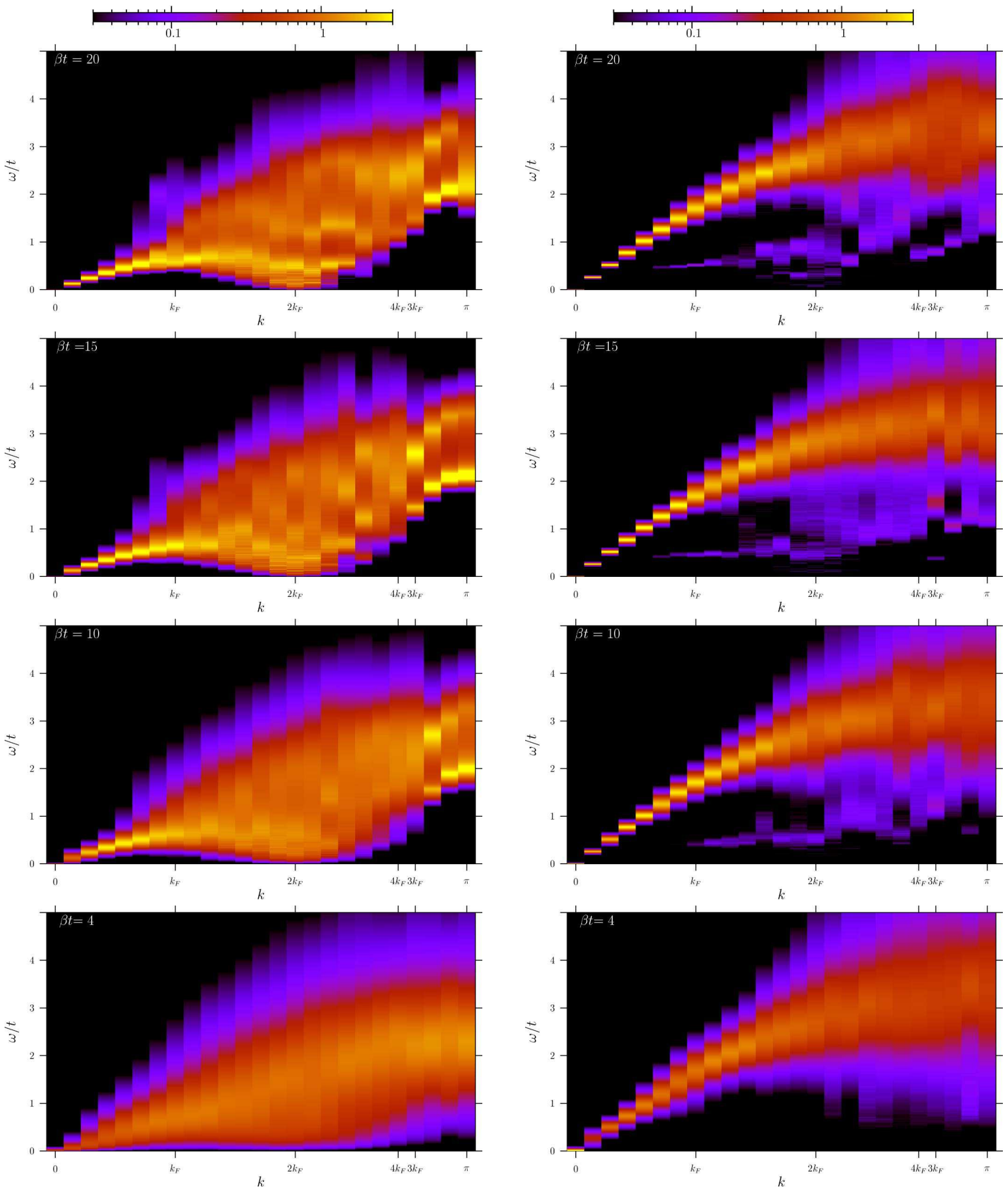

FIG. 3: Dynamical spin-spin correlations as a function of temperature on a logarithmic intensity scale. The inverse temperatures from top to bottom read: $\beta t=20,15,10,4$.

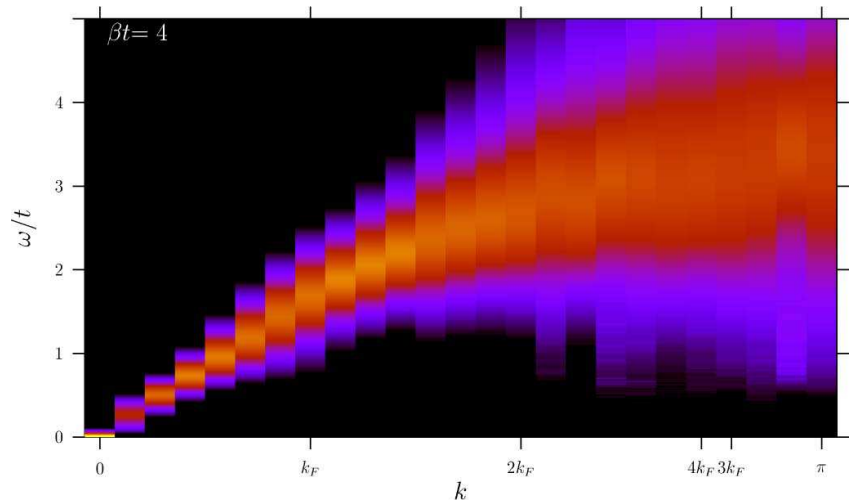

FIG. 4: Dynamical charge-charge correlations as a function of temperature on a logarithmic intensity scale. The inverse temperatures from top to bottom read: $\beta t=20,15,10,4$. 


\section{A. Dynamical spin and charge correlation functions.}

To investigate the spin and charge dynamics we consider the dynamical susceptibility

$$
\chi_{c}(k, \omega)=-i \int_{0}^{\infty} \mathrm{d} t e^{i \omega t}\left\langle\left[O_{c}(k, t), O_{c}(-k, 0)\right]\right\rangle
$$

where $O_{c}(\vec{k})=\frac{1}{\sqrt{N}} \sum_{\vec{r}} e^{i \vec{k} \cdot \vec{r}}\left(n_{\vec{r}, \uparrow} \pm n_{\vec{r}, \downarrow}\right)$. Fig. 2 plots the static spin and charge susceptibilities, $\chi_{s}(\vec{k}, \omega=0)$ as a function of temperature. Those quantities measure correlation lengths and allow us to identify crossover energy scales below which spin and charge fluctuations grow as a function of decreasing temperature. Since one dimensional systems are critical at $T=0$ both $2 k_{f}$ spin and charge static susceptibilities diverge at $T=0$. As apparent from Fig. 2 the crossover scale marking the growth of $2 k_{f}$ spin fluctuations is given by $T_{J} \simeq t / 10$. A similar energy scale for the $2 k_{f}$ charge fluctuations can be read off Fig. 2] However the magnitude of the signal, and hence the amplitude of the $2 k_{f}$ charge modulation is substantially smaller than in the spin sector. The Luttinger liquid parameter, $K_{0}$ of the Hubbard model is bounded by $1 / 2<K_{\rho}<1$ [10]. Hence $4 k_{f}$ charge-charge correlations which decay as $r^{-4 K_{\rho}}$ are very much suppressed in comparison to $2 k_{f}$ charge fluctuations which decay as $r^{-1-K_{\rho}}$. This stands in accordance with the data of Fig. 2 and no divergence in the $4 k_{f}$ charge susceptibility is expected.

Having pinned down energy scales we now consider the dynamical spin and charge structure factors:

$$
S_{c}(\vec{k}, \omega)=\frac{1}{1-e^{-\beta \omega}} \operatorname{Im} \chi_{s}(\vec{k}, \omega) .
$$

Fig. 3 plots the dynamical spin structure factor as a function of temperature. As apparent below the crossover scale $T_{J}$ the two spinon continuum of excitations with gapless excitations at $2 k_{F}$ is clearly visible. Furthermore, below $T_{J}$, a well defined spin velocity can be read off the data yielding $v_{s} / t \simeq 1$. This result compares favorably with the zero temperature results of Ref. [11]. Hence, in the spin sector $T_{J}$ marks the temperature scale below which the the overall features of the zero temperature dynamical spin structure factor become apparent.

The dynamical charge structure factor is plotted as a function of temperature in Fig. 4 Again, below $T_{J}$, one can read off the charge velocity, $v_{c} \simeq 1.9$ which favorably compares with the zero temperature data of [11]. Given the small amplitude of the $2 k_{f}$ charge fluctuations, we are unable to reliably pin down the expected gapless excitations at $2 k_{f}$ as well as at $4 k_{f}$.

\section{B. Single particle excitation spectrum}

Our major interest here is to study the temperature dependence of the single particle spectral function and

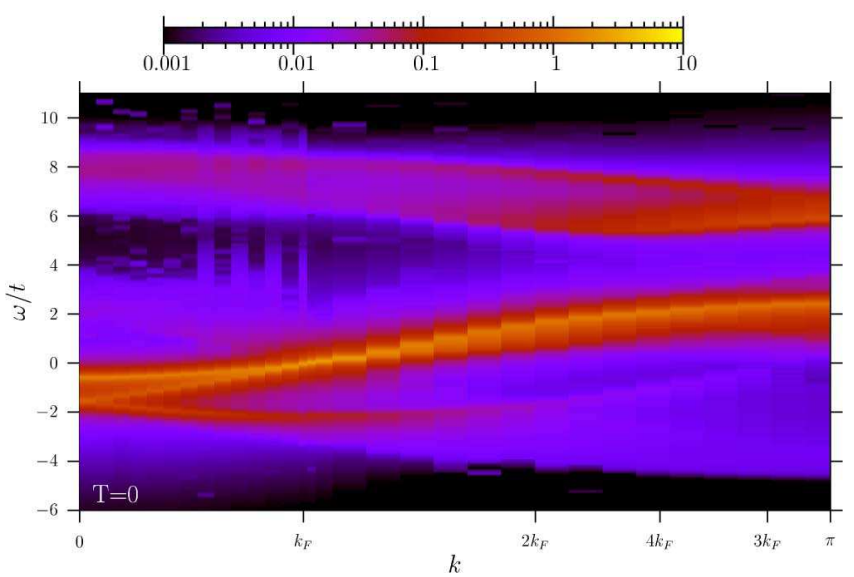

FIG. 5: Single particle excitation spectrum for $T=0$ shown as gray scale plot with a logarithmic intensity scale. These data stem from the DDMRG calculations of $\mathrm{H}$. Benthien et al. 3]

compare to the experiments of Ref. [5]. At the lowest temperatures considered in Ref. [5], the photoemission results compare favorably with $T=0$ DDMRG calculations of Ref. [3]. In the photoemission spectra one can identify a spinon branch, a holon branch as well a holon shadow band. Those features compare well with the zero temperature DDMRG data shown in Fig. [5. Let us concentrate on $\omega / t<0$ relevant for comparison with photoemission. In the vicinity of the Fermi wave vector and at low energies one clearly observes two features (branchcuts) dispersing linearly with velocities $v_{s}$ (spinon) and $v_{c}$ (holon). Those velocities stand in good agreement with those determined by our analysis of the spin and charge dynamical structure factors. Furthermore, and at low energies one can identify a feature at $3 k_{f}$ which merges at $k=0$ with the holon branch. Following Ref. 2] one notes that this feature has the same dispersion relation as the holon branch but shifted by $2 k_{f}$. Hence the interpretation of a shadow holon branch which stems from a holon scattering off a $2 k_{f}$ spin excitation. This is very reminiscent of shadow bands in spin-density wave approaches of antiferromagnetic Mott insulators.

The finite temperature spectra we have obtained with the QMC are presented in Fig. [6] The question we wish to address is at which temperature scale do the features of the $T=0$ data become apparent. One can observe a clear spinon branch for the different temperatures except at $\beta t=2$ where spectrum is washed out. The holon shadow band can also be identified at least in the region of wave vectors where $0 \leq k \leq k_{F}$. As can be seen in the DDMRG $T=0$ spectrum, the intensity of the holon shadow band rapidly decreases for larger wave vectors making it very hard to retrace this feature in our spectra. Another difficulty arises regarding the holon branch in our QMC spectra. To analyze our data we use the stochastic MEM, with its well-known difficulties to re- 

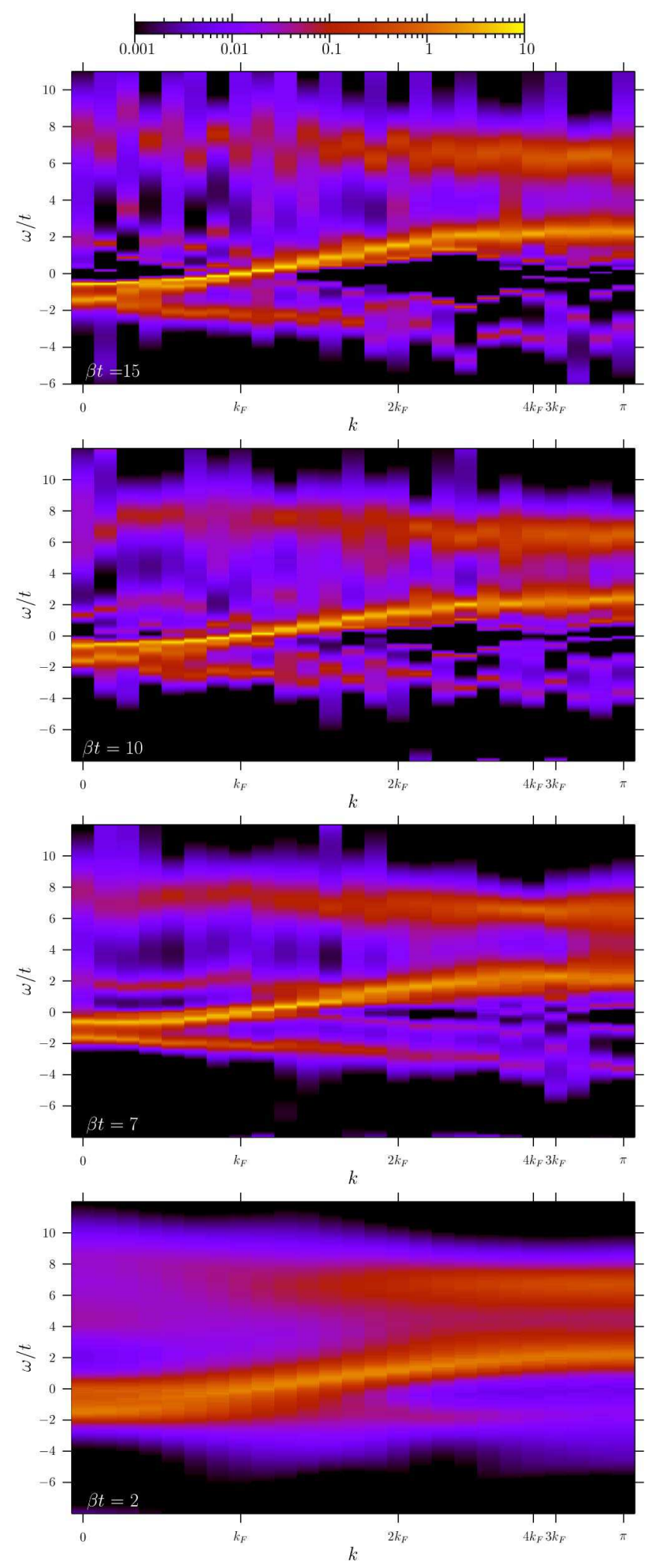

FIG. 6: Single particle excitation spectrum as a function of temperature on a logarithmic intensity plot. From top to bottom: $\beta t=15,10,7,2$.
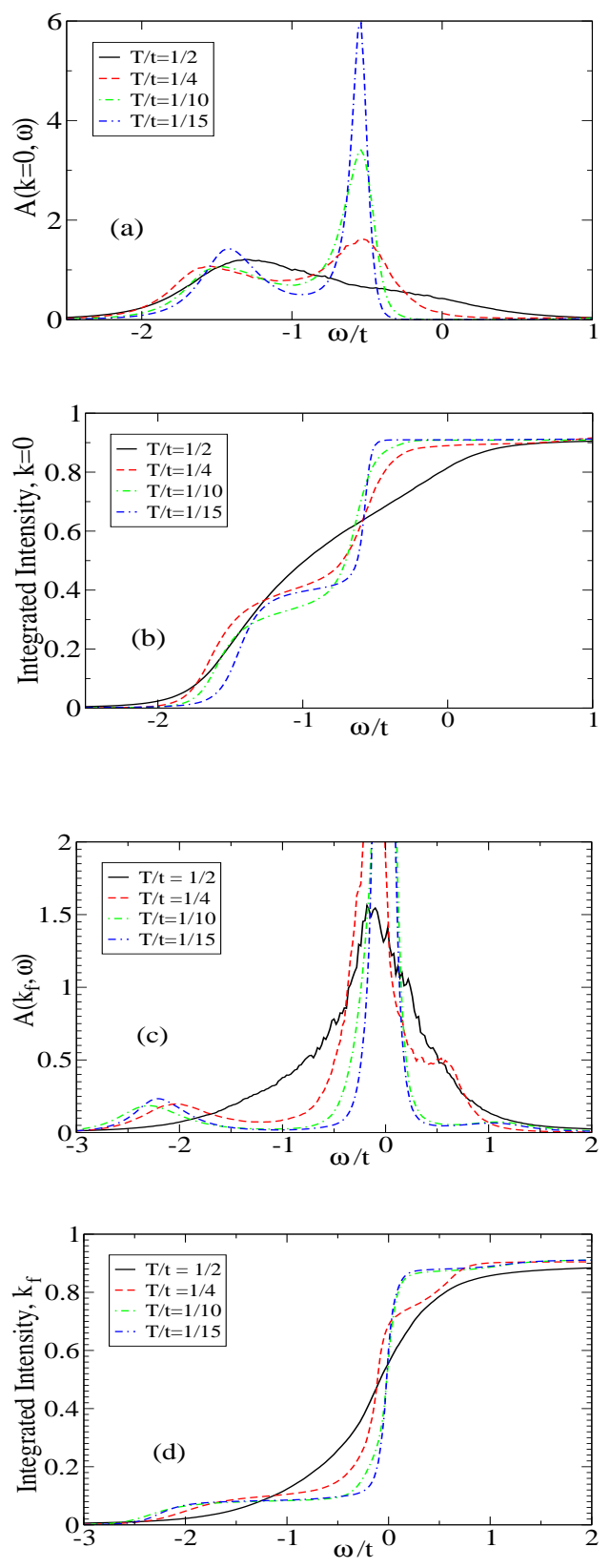

FIG. 7: Temperature dependence of spectral functions at (a) $k=0$ and (c) $k=k_{f}$. Integrated spectral weight for (b) $k=0$ and (c) $k=k_{f}$.

solve two peaks close in energy. Nevertheless, at our lowest presented temperature $\beta t=15$, a holon branch can be identified. Hence, the finite temperature results stand in agreement with the statement that below the spin scale $T_{J}$, the gross features of the zero temperature results are apparent.

The ARPES measurements of Ref. [5] point towards spectral weight transfer in the temperature range $60 K<$ $T<260 K$. At the Fermi wave vector and as a function of decreasing temperature spectral weight is transfered from higher $(\simeq 0.7 \mathrm{eV})$ to lower $(\simeq 0.1 \mathrm{eV})$ excita- 
tion energies. The numerical simulations do indeed show spectral weight transfer however at a temperature scale $T>T_{J}$. Figs. 7 plots $A(k, \omega)$ as a function of temperature for $k=0$ and $k=k_{f}$. Upon inspection of the data, one observes that at high temperatures $(\beta t=2)$, the spectral weight is dominantly located at frequency of the holon, $\omega / t \simeq-1.5$ for $k=0$ and $\omega \simeq 0$ at $k=k_{f}$. As temperature is lowered thereby generating short ranged $2 k_{f}$ spin fluctuations, spectral weight is shifted over an energy scale set by $t$ to form the spin related features. At $k=0$, this corresponds to the spinon at $\omega / t \simeq-0.5$ and at $k=k_{f}$ to the holon-shadow band at $\omega / t \simeq-2$. For $T<T_{J}$ and within the limitations of the stochastic analytical continuation, the data shows no further shift of spectral weight.

\section{CONCLUSION}

We have computed the temperature dependence of the single particle spectral function for the one-dimensional Hubbard model, for a parameter range which has been proposed [5] for the modeling of the TCNQ band in TTFTCNQ organics; $U / t=4.9, t=0.4 \mathrm{eV}$ and $n=0.59$. This parameter set reproduces well the overall features of the photoemission spectra at $T=60 K$ above the Peierls temperature. For this parameter set we have identified a magnetic energy scale $T_{J} \simeq 0.1 t$ below which $2 k_{f}$ spin fluctuations are enhanced as a function of decreasing temperature. For $T<T_{J}$ the overall features of the $T=0$ spectral function are apparent. In particular no shift in spectral weight between holon, holon-shadow and spinon branches is observed below this temperature scale. On the other hand, for $T>T_{J}$ spectral weight transfer over an energy scale set by $t$ is observed. Very similar conclusions have been reached for the half-filled Hubbard model [12]. With $t=0.4 \mathrm{eV}$ we obtain a magnetic scale $T_{J} \simeq 400 \mathrm{~K}$ and hence we are unable to account for the spectral weight transfer observed in the photoemission experiments in the temperature range $60 K<T<260 K$ [5].

Assuming that a pure electronic model is valid to account for the temperature dependence of the spectral function, other parameter sets are required to understand the experimental data. The aim is to keep the low temperature spectral function similar to that observed in this work since it compares well with the low temperature photoemission data, but to reduce the spin scale $T_{J}$. X-ray scattering experiments of Ref. [13] suggest that both $4 k_{f}$ and to $2 k_{f}$ charge fluctuations are present at low temperatures and that above $150 K$ only $4 k_{f}$ scattering is present. To model such dominant $4 k_{f}$ fluctuations one requires a Luttinger liquid parameter $K_{\rho}<1 / 2$ [10]. Since the Hubbard model has $1 / 2<K_{\rho}<1$ additional terms such as a nearest neighbor Coulomb repulsion $V$ is required. However, we expect that $V$-terms in the Hamiltonian will enhance the overall low temperature band width. This band-width problem could be corrected by reducing the value of the hopping matrix element to it's bulk value, $t \sim 0.2 \mathrm{eV}$, as inferred from DFT calculations [5]. In turn this would enhance the value of $U / t$ and hence reduce the value of $T_{J}$. Further simulations are required to confirm this point of view.

The issue of coupling to the lattice is still open. In particular since the system is close to a Peierls transition, it is not clear that phonons can be omitted. Furthermore the line-shapes of model calculations at low temperature are much sharper than the experimentally observed. Coupling to the lattice, could account for this broadening.

Acknowledgments. Part of the calculations presented here were carried out on the IBM p690 cluster of the NIC in Jülich. We would like to thank this institution for allocation of CPU time. We have greatly profited from discussions with R. Claessen and would like to thank E. Jeckelmann for sending us the DDMRG results. Similar issues concerning the temperature behavior of the spectral function for TTF-TCNQ have been addressed by N. Bulut, H. Matsueda, T. Tohoyama and S. Maekawa. We would like to thank those authors for sending us a version of their manuscript prior to publication. Financial support from the Centre de Coopération Universitaire Franco-Bavarois (CCUFB-BFHZ) is acknowledge.
[1] T. Giamarchi, Quantum physics in one dimension (Clarendon Press, Oxford, 2004), iSBN 019852500 1.

[2] K. Penc, K. Hallberg, F. Mila, and H. Shiba, Phys. Rev. Lett 77, 1390 (1996).

[3] H. Benthien, F. Gebhard, and E. Jeckelmann, Phys. Rev. Lett. 69, 256401 (2004).

[4] R. Preuss, A. Muramatsu, W. von der Linden, P. Dieterich, F. Assaad, and W. Hanke, Phys. Rev. Lett. 73, 732 (1994).

[5] M. Sing, U. Schwingenschlögl, R. Claessen, P. Blaha, J. M. P. Carmelo, L. M. Martelo, P. D. Sacramento, M.
Dressel, and C. S. Jacobsen, Phys. Rev. B 68, 125111 (2003).

[6] F. F. Assaad, in Lecture notes of the Winter School on Quantum Simulations of Complex Many-Body Systems :From Theory to Algorithms., edited by J. Grotendorst, D. Marx, and A. Muramatsu. (Publication Series of the John von Neumann Institute for Computing., ADDRESS, 2002), Vol. NIC series Vol. 10., pp. 99-155.

[7] K. S. D. Beach, cond-mat/0403055 (2004).

[8] M. Jarrell and J. Gubernatis, Physics Reports 269, 133 (1996).

[9] W. von der Linden, Applied Physics A 60, 155 (1995). 
[10] H. Schulz, Phys. Rev. Lett 64, 2831 (1990).

[11] H. Schulz, cond-mat/9503150.

[12] H. Matsueda, N. Bulut, T. Tohyama, and S. Maekawa, Phys. Rev. B 72, 075136 (2005).
[13] J. Pouget, S. K. Khanna, F. Denoyer, R. Comès, A. F. Garito, and A. J. Heeger, Phys. Rev. Lett. 37, 437 (1976). 